\title{
Single 20-Second Acquisition of Deep-Inspiration Breath-Hold PET/CT: Clinical Feasibility for Lung Cancer
}

Tatsuo Torizuka ${ }^{1}$, Yasuo Tanizaki ${ }^{2}$, Toshihiko $\mathrm{Kanno}^{2}$, Masami Futatsubashi ${ }^{3}$, Etsuji Yoshikawa ${ }^{3}$, Hiroyuki Okada ${ }^{3}$, and Yasuomi Ouchi ${ }^{4}$

${ }^{I}$ Research Center for Child Mental Development, Hamamatsu University School of Medicine, Hamamatsu, Japan;

${ }^{2}$ Positron Medical Center, Hamamatsu Medical Center, Hamamatsu, Japan; ${ }^{3}$ Central Research Laboratory,

Hamamatsu Photonics K.K., Hamamatsu, Japan; and ${ }^{4}$ Molecular Imaging Frontier Research Center, Hamamatsu University

School of Medicine, Hamamatsu, Japan

This study was designed to compare tumor ${ }^{18} \mathrm{~F}-\mathrm{FDG}$ uptake between a single 20 -s acquisition of deep-inspiration breath-hold PET/CT and free-breathing PET/CT for lung cancer. Methods: Before the clinical study, a phantom study was performed to determine the optimum breath-hold time for the PET scan. We studied 47 patients with lung cancer who underwent free-breathing PET/CT with the standard clinical protocol, followed by deepinspiration breath-hold PET/CT of the thorax. In breath-hold $\mathrm{PET} / \mathrm{CT}$, the patients were asked to hold their breath in deep inspiration for $10 \mathrm{~s}$ during the CT scan and for $20 \mathrm{~s}$ during the PET scan. Maximum tumor ${ }^{18} \mathrm{~F}-\mathrm{FDG}$ standardized uptake value (SUVmax) was measured in free-breathing PET and breathhold PET, and the percentage difference between these 2 values was calculated. Results: Breath-hold PET showed a significant increase in SUVmax, as compared with free-breathing PET (8.26 \pm 4.59 vs. $11.25 \pm 7.24, P<0.0001)$. The mean difference in SUVmax was $39.5 \% \pm 43.4 \%$, and the range was $2.9 \%-248.3 \%$. The difference in SUVmax was significant when compared between tumors in the upper lung $(n=22)$ and tumors in the lower lung $(n=25)(24.4 \% \pm 17.7 \%$ vs. $52.9 \% \pm 54.3 \%, P=0.0077)$. The mean tumor size of the group with a high SUVmax difference ( $n=13$ ) was significantly smaller than that of the group with a low SUVmax difference $(n=34)(2.45 \pm 0.87 \mathrm{~cm}$ vs. $3.21 \pm 1.22 \mathrm{~cm}$, $P=0.043$ ), using a cutoff of $39.5 \%$. Conclusion: The single $20-\mathrm{s}$ acquisition of breath-hold PET/CT enabled more precise measurement of SUVmax, especially in the lower lung field and for small tumors, which may be affected by respiratory motion. This technique is feasible in the clinical setting and requires only a minor increase in examination time.

Key Words: oncology; PET; PET/CT; breath hold; FDG PET/CT; lung cancer; SUVmax

J Nucl Med 2009; 50:1579-1584

DOI: 10.2967/jnumed.109.064246

Received Mar. 14, 2009; revision accepted Jun. 29, 2009.

For correspondence or reprints contact: Tatsuo Torizuka, Research Center for Child Mental Development, Hamamatsu University School of Medicine, 1-20-1 Handayama, Higashi-ku, Hamamatsu 431-3192, Japan. E-mail: tatsuo@hama-med.ac.jp

COPYRIGHT @ 2009 by the Society of Nuclear Medicine, Inc.
$\mathbf{T}$ he value of combined PET/CT for diagnosis and staging of malignant lesions has been well recognized. Although the fusion of PET and CT images significantly improves both sensitivity and specificity in the detection of diseases, image misregistration may occur in the chest and abdomen because of motion artifacts caused by respiration $(1-3)$. Although the CT scan is acquired in a few seconds, capturing the chest at a single phase of a single respiratory cycle, the emission PET scan is acquired over many minutes and averages many respiratory cycles. As a result, the 2 datasets frequently do not overlie each other. Additionally, respiratory motion blurs PET images, degrades contrast, overestimates tumor volume, and increases variability in standardized uptake value (SUV).

In recent years, several methods of compensating for respiratory motion have been applied to PET/CT acquisitions (4-9). Four-dimensional (4D) PET and CT is technically feasible and has been reported to improve coregistration of PET and CT lesions; however, a major drawback of 4D $\mathrm{CT}$ is the increased radiation dose to patients $(7,8)$. Deepinspiration breath-hold PET/CT has been used as an alternative to $4 \mathrm{D} \mathrm{PET} / \mathrm{CT}$, with a real-time position management system used to monitor respiratory motion and to obtain acquisitions $(5,6)$. This method principally overcomes the limitation of increased radiation dose in 4D PET/CT. In this technique, the PET scan is matched to the CT scan through summing of multiple acquisitions in nine 20-s independent frames obtained at end-inspiration apnea. Although this technique, compared with non-motion-corrected PET/CT, increases lesion SUV and improves spatial matching between PET and CT images, the PET acquisition time needs to be increased to obtain good-quality PET images.

Recently, Kawano et al. studied the single acquisition of deep-inspiration breath-hold PET/CT (10). The patients were instructed to hold their breath during maximal inspiration for as long as possible during the PET scan. Although the investigators concluded that this technique may be feasible 
for SUV analysis and tumor registration in clinical PET/CT, the mean breath-hold time was $54.2 \pm 20.8 \mathrm{~s}$, and the range was 30-125 s. Requiring deep-inspiration apnea for such a long time would not seem acceptable for uncooperative or sick patients. In the present study, we instructed patients to hold their breath in deep inspiration for $20 \mathrm{~s}$ during a single PET scan and we compared tumor ${ }^{18}$ F-FDG uptake between breath-hold PET/CT and free-breathing PET/CT. Before the clinical study, we performed a phantom study to determine the optimum breath-hold time for the PET scan.

\section{MATERIALS AND METHODS}

\section{Phantom Study}

For the phantom study, we used an International Electrotechnical Commission body phantom set corresponding to the NU 2-2001 standard published by the National Electrical Manufacturers Association. The phantom set consisted of a torso cavity, a removable lung insert, and 6 spheres (inner diameters: 10, 13, $17,22,28$, and $37 \mathrm{~mm}$ ). The torso cavity was filled with water, and the 6 spheres were filled with ${ }^{18} \mathrm{~F}$-FDG solutions of the same radioactivity concentration $(20 \mathrm{kBq} / \mathrm{mL})$. PET/CT was performed at a single bed position. PET images $(n=4)$ were acquired for $120 \mathrm{~s}$ consecutively in 5 -s frames for $50 \mathrm{~s}$ and then in 10-s frames for $70 \mathrm{~s}$, using the list-mode dynamic collection method. The acquisition time of $120 \mathrm{~s}$ corresponded to the standard clinical protocol described below. Image reconstruction was performed using the same reconstruction parameters as for the clinical study. Regions of interest were placed over all spheres, and the maximum radioactivity was measured. The mean and $\mathrm{SD}$ of the maximum radioactivity for the 4 images were calculated at the 10-, 15-, 20-, 25-, 30-, 60-, and 120-s acquisition times. The coefficient of variation $(\mathrm{CV})$ was defined as follows: $\mathrm{CV}=\mathrm{SD}$ / mean $\times 100$. Furthermore, the percentage of maximum radioactivity relative to that at $120 \mathrm{~s}$ was calculated at each acquisition time.

Tables 1 and 2 show the results of the phantom study. The CV tended to be higher in smaller spheres and increased with shorter acquisitions (Table 1). The mean and SD of the percentage of radioactivity for the 4 images are shown in Table 2 . When the acquisition time was $20 \mathrm{~s}$ or longer, the mean percentage was within $100 \% \pm 5 \%(95 \%-105 \%)$ for all spheres larger than 13 $\mathrm{mm}$ in inner diameter. From these results of the phantom study, we determined that a 20-s frame would be used for acquisition of the breath-hold PET portion of the clinical PET/CT study.

\section{Patient Data}

Forty-seven consecutive patients with lung cancer were enrolled in the ${ }^{18} \mathrm{~F}$-FDG PET/CT study. This group comprised 37 men and 10 women with a mean age of $68 \mathrm{y}$ (range, 51-89y). On the basis of the phantom study, patients with lung tumors smaller than $13 \mathrm{~mm}$ were excluded. We also excluded lung tumors showing weak ${ }^{18} \mathrm{~F}-\mathrm{FDG}$ uptake (SUVmax $<1.5$ ) on free-breathing PET. All patients provided written informed consent for participation in the PET study, which was approved by our institutional review board.

\section{PET/CT Acquisition}

All patients fasted for at least $5 \mathrm{~h}$ before the PET studies. Serum glucose levels measured at the time of ${ }^{18} \mathrm{~F}$-FDG injections were less than $150 \mathrm{mg} / \mathrm{dL}$ in all patients. The PET/CT scanner was a Biograph Sensation 16 (Siemens Medical Solutions). The PET component of this scanner consists of 24 detector rings of lutetium oxyorthosilicate and is a 3-dimensions-only tomograph. The axial and transverse fields of view are 16.2 and $58.5 \mathrm{~cm}$, respectively. The transverse resolution is $6.5 \mathrm{~mm}$ and the axial resolution 6.0 $\mathrm{mm}$-both at a radius of $1 \mathrm{~cm}$. The CT component is a 16-slice spiral scanner with a variable slice thickness of 0.6-10.0 $\mathrm{mm}$ and a $50-\mathrm{cm}$ transverse field of view that can be extended to $70 \mathrm{~cm}$ by means of a fitting algorithm. This scanner is equipped with a research package for list-mode acquisition (Siemens Medical Solutions).

Each of the patients was injected intravenously with $5 \mathrm{MBq}$ of ${ }^{18}$ F-FDG per kilogram of body weight and then remained recumbent during an uptake phase of approximately $60 \mathrm{~min}$. A clinical PET/CT session was performed according to the standard clinical protocol at our institution. CT data were acquired in helical mode, and then PET was performed for the corresponding CT axial length at a rate of $2 \mathrm{~min}$ per field of view. These CT and PET scans were acquired with the patients under free breathing, with no voice instructions.

The clinical PET/CT session was followed by the breath-hold PET/CT study in the same position (supine, arms above head). CT data were acquired in the same setting as for the standard clinical protocol. The real-time position management system (AZ-733V; Anzai Medical Co. Ltd.) was used in the amplitude-gating mode to monitor the patient's respiratory motion. The patient was instructed to breathe deeply and then hold the breath for approximately $10 \mathrm{~s}$ during the CT acquisition of the lung field, including a target lesion. PET data for a single field of view were then acquired in a 20-s frame under a deep-inspiration breath-hold. If the patient failed to hold the breath in the same amplitude as for the CT acquisition, additional scans were required. Additional scans were required for $15 \%-20 \%$ of the patients, and only 1 scan was repeated for most of those patients. The entire time from patient setup to completion of the scan was 4-5 min on average.

\section{PET/CT Reconstruction and Analysis}

PET image reconstruction was performed with Fourier rebinning and attenuation-weighted ordered-subsets expectation max-

\begin{tabular}{|c|c|c|c|c|c|c|c|}
\hline \multirow[b]{2}{*}{ Sphere size (mm) } & \multicolumn{7}{|c|}{ Acquisition time (s) } \\
\hline & 10 & 15 & 20 & 25 & 30 & 60 & 120 \\
\hline 10 & 43.75 & 47.34 & 43.81 & 44.06 & 41.58 & 37.02 & 35.65 \\
\hline 13 & 42.97 & 39.46 & 35.50 & 36.47 & 35.64 & 31.61 & 30.65 \\
\hline 17 & 26.32 & 24.54 & 23.95 & 21.03 & 21.49 & 19.19 & 19.52 \\
\hline 22 & 11.45 & 8.71 & 8.06 & 8.33 & 7.40 & 8.33 & 7.27 \\
\hline 28 & 4.64 & 4.50 & 3.92 & 2.48 & 2.02 & 2.38 & 1.37 \\
\hline 37 & 6.99 & 3.53 & 2.87 & 2.74 & 3.51 & 1.73 & 1.30 \\
\hline
\end{tabular}


TABLE 2. Results of Phantom Study: Percentage of Maximum Radioactivity Relative to That at 120 -Second Acquisition Time $(n=4)$

\begin{tabular}{|c|c|c|c|c|c|c|c|}
\hline \multirow[b]{2}{*}{ Sphere size $(\mathrm{mm})$} & \multirow[b]{2}{*}{ Parameter } & \multicolumn{6}{|c|}{ Acquisition time (s) } \\
\hline & & 10 & 15 & 20 & 25 & 30 & 60 \\
\hline \multirow[t]{2}{*}{10} & Mean & 50.11 & 75.06 & 80.67 & 87.42 & 89.98 & 96.97 \\
\hline & SD & 9.04 & 8.62 & 2.97 & 2.72 & 2.13 & 5.10 \\
\hline \multirow[t]{2}{*}{13} & Mean & 75.02 & 91.59 & 98.08 & 97.81 & 98.98 & 96.75 \\
\hline & $\mathrm{SD}$ & 3.75 & 3.23 & 2.61 & 1.42 & 3.93 & 2.07 \\
\hline \multirow[t]{2}{*}{17} & Mean & 88.98 & 95.97 & 97.10 & 99.28 & 98.73 & 100.20 \\
\hline & $\mathrm{SD}$ & 4.01 & 1.31 & 1.83 & 0.66 & 1.75 & 1.62 \\
\hline \multirow[t]{2}{*}{22} & Mean & 96.81 & 97.79 & 99.40 & 95.69 & 96.01 & 98.76 \\
\hline & $\mathrm{SD}$ & 1.79 & 3.51 & 3.40 & 2.17 & 2.25 & 1.67 \\
\hline \multirow[t]{2}{*}{28} & Mean & 97.31 & 100.42 & 99.34 & 100.35 & 100.43 & 101.86 \\
\hline & SD & 2.65 & 1.32 & 1.85 & 2.03 & 2.82 & 2.15 \\
\hline \multirow[t]{2}{*}{37} & Mean & 104.43 & 99.99 & 100.99 & 100.86 & 102.62 & 100.86 \\
\hline & SD & 3.21 & 2.93 & 1.36 & 2.50 & 3.26 & 0.68 \\
\hline
\end{tabular}

imization using CT-based attenuation correction. The same clinical reconstruction parameters were used for free-breathing PET images and breath-hold PET images (2 iterations, 8 subsets, and 5-mm gaussian filter). All PET/CT datasets were displayed on a Leonardo workstation (e-soft; Siemens).

For each lesion, the maximum SUV (SUVmax) was obtained for both free-breathing PET images and breath-hold PET images. The maximum diameter of each lesion was measured from the breath-hold CT image, using a lung window (window, 1,200 Hounsfield units; level, -600 Hounsfield units). The percentage difference between free-breathing SUVmax and breath-hold SUVmax was defined as follows: (breath-hold SUVmax - freebreathing SUVmax)/free-breathing SUVmax $\times 100$.

\section{Statistical Analysis}

The nonparametric Mann-Whitney $U$ test was applied to compare parameters between the 2 groups (upper-lung and lower-lung

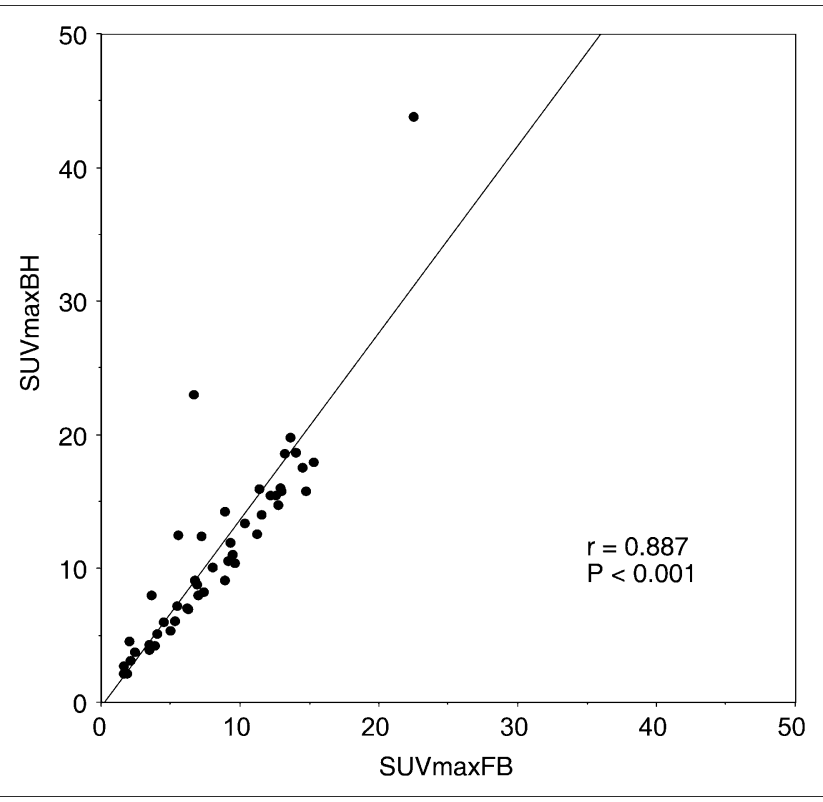

FIGURE 1. Graph showing significant correlation between free-breathing SUVmax (SUVmaxFB) and breath-hold SUV$\max (\mathrm{SUVmaxBH})$. groups; groups with high and low differences in SUVmax). A $P$ value of less than 0.05 was considered significant.

\section{RESULTS}

We studied 47 patients with histologically proven lung cancer. The mean maximum tumor diameter was $3.00 \pm 1.18$ $\mathrm{cm}$ (range, $1.6-6.0 \mathrm{~cm}$ ). The effective radiation doses to patients from whole-body PET/CT and breath-hold CT were 7.0-7.8 and 1.1-1.3 $\mathrm{mSv}$, respectively. The mean freebreathing SUVmax and breath-hold SUVmax were $8.26 \pm$ 4.59 and $11.25 \pm 7.24$, respectively $(P<0.0001)$. There was a significant correlation between these 2 values $(r=0.887$, $P<0.001$ ) (Fig. 1). The mean difference in SUVmax was $39.5 \% \pm 43.4 \%$, and the range was $2.9 \%-248.3 \%$.

These 47 lung lesions were divided into the following 2 groups. The upper-lung group included 22 lesions of the upper lobe, and the lower-lung group included 24 lesions of the lower lobe and 1 lesion of the right middle lobe. Table 3 compares the 2 groups for tumor size, free-breathing SUVmax, breath-hold SUVmax, and percentage difference in SUVmax. There was no significant difference in tumor size between the upper-lung and lower-lung groups $(2.92 \pm 1.26$ cm vs. $3.04 \pm 1.12 \mathrm{~cm}, P=0.347$ ). Although no significant difference in free-breathing SUVmax was observed between the 2 groups $(7.05 \pm 3.93$ vs. $9.33 \pm 4.93, P=0.101)$, the breath-hold SUVmax of the lower-lung group was significantly higher than that of the upper-lung group (13.62 \pm 8.26

\begin{tabular}{|c|c|c|c|}
\hline Parameter & $\begin{array}{l}\text { Upper lung } \\
(n=22)\end{array}$ & $\begin{array}{l}\text { Lower lung } \\
\qquad(n=25)\end{array}$ & $P$ \\
\hline Tumor size $(\mathrm{cm})$ & $2.92 \pm 1.26$ & $3.04 \pm 1.12$ & 0.347 \\
\hline $\begin{array}{l}\text { Free-breathing } \\
\text { SUVmax }\end{array}$ & $7.05 \pm 3.93$ & $9.33 \pm 4.93$ & 0.101 \\
\hline Breath-hold SUVmax & $8.57 \pm 4.76$ & $13.62 \pm 8.26$ & 0.015 \\
\hline $\begin{array}{l}\text { Difference in } \\
\text { SUVmax (\%) }\end{array}$ & $24.4 \pm 17.7$ & $52.9 \pm 54.3$ & 0.0077 \\
\hline
\end{tabular}


FIGURE 2. CT (A), free-breathing PET/ CT (B), and breath-hold PET/CT (C) scans of patient with lung cancer in left upper lobe (adenocarcinoma, $16 \mathrm{~mm}$ ). Free-breathing SUVmax $=4.93$, breathhold SUVmax $=5.40$, and difference in SUVmax $=9.5 \%$. Free-breathing PET/ CT showed minimal misregistration between PET and CT.
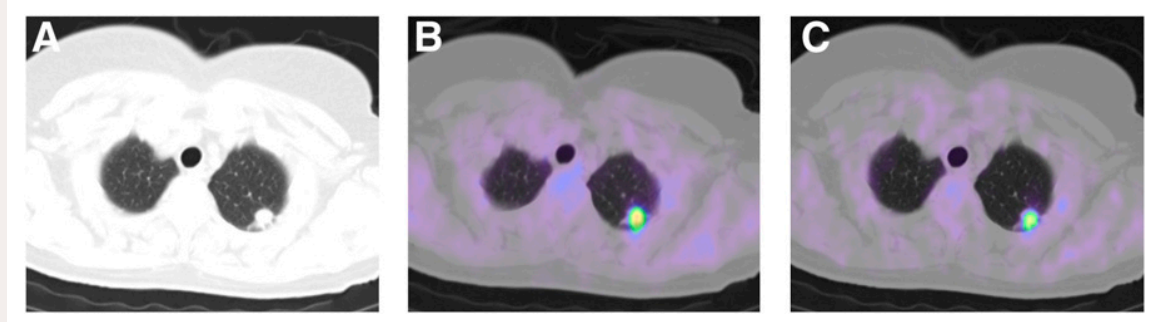

vs. $8.57 \pm 4.76, P=0.015)$. In addition, the lower-lung group showed a significantly higher difference in SUVmax than did the upper-lung group $(52.9 \% \pm 54.3 \%$ vs. $24.4 \% \pm$ $17.7 \%, P=0.0077$ ) (Figs. 2-4).

When the 47 lesions were divided into groups with high or low differences in SUVmax, using a cutoff of 39.5\%, 13 lesions were in the high-difference group and 34 lesions in the low-difference group. Tumor size in the high-difference group was significantly smaller than that in the low-difference group ( $2.45 \pm 0.87 \mathrm{~cm}$ vs. $3.21 \pm 1.22 \mathrm{~cm}, P=0.043)$, whereas no significant difference between these 2 groups was observed in free-breathing SUVmax (7.74 \pm 6.12 vs. $8.46 \pm$ $3.95, P=0.373)$ or breath-hold SUVmax $(14.06 \pm 11.21$ vs. $10.18 \pm 4.80, P=0.379$ ) (Table 4). The same analysis was performed for the upper-lung and lower-lung groups, separately. In the upper-lung group, there was no significant difference in tumor size between the group with a high difference in SUVmax $(n=4)$ and the group with a low difference in SUVmax $(n=18)(2.78 \pm 1.36 \mathrm{~cm}$ vs. $2.96 \pm$ $1.28 \mathrm{~cm}, P=0.831$ ) (Table 5). In the lower-lung group, however, the 9 tumors with a high difference in SUVmax were significantly smaller than the 16 tumors with a low difference in SUVmax $(2.31 \pm 0.61 \mathrm{~cm}$ vs. $3.51 \pm 1.12 \mathrm{~cm}$, $P=0.0083$ ) (Table 6). For free-breathing SUVmax and breath-hold SUVmax, no significant difference was found between the group with a high difference in SUVmax and the group with a low difference in SUVmax in either the upperor the lower-lung group.

\section{DISCUSSION}

In this study, we determined a procedure to acquire single 20-s acquisitions of deep-inspiration breath-hold PET/CT and found that this technique enabled more precise measurement of the SUVmax of lung tumors. Compared with free-breathing PET/CT, breath-hold PET/CT increased the mean SUVmax by $39.5 \%$. This method is clinically feasible and requires only a minor increase in examination time.

Respiratory motion spreads tracer activity within a given lesion over a larger area and thus underestimates the true activity concentration. It is desirable to eliminate these artifacts and develop an imaging technique that allows accurate PET/CT alignment in the thorax. 4D PET/CT protocols have been developed to reduce respiratory motion artifacts but have the shortcoming of long acquisition and postprocessing times $(7,8)$. During 4D PET/CT, patients must maintain a regular respiratory pattern for an extended time- a requirement that may not be acceptable for patients with underlying lung disease. In addition, a major drawback of 4D CT is the increased radiation dose to the patient. A 4D CT scan has been reported to deliver a dose 4 times that of a diagnostic chest scan (7).

FIGURE 3. Transaxial free-breathing $\mathrm{CT}$, sagittal free-breathing PET, and sagittal free-breathing PET/CT scans (A), as well as transaxial breath-hold CT, sagittal breath-hold PET, and sagittal breath-hold PET/CT scans (B), of patient with lung cancer in right lower lobe (adenocarcinoma, $23 \mathrm{~mm}$ ). Freebreathing SUVmax $=3.60$, breath-hold SUVmax $=8.06$, and difference in SUVmax $=123.9 \%$. Transaxial CT slices correspond to middle of lesion for both PET studies. In free-breathing $\mathrm{PET} / \mathrm{CT}$, misregistration between PET and CT was apparent. Breath-hold PET/ CT improved coregistration and lesion localization.
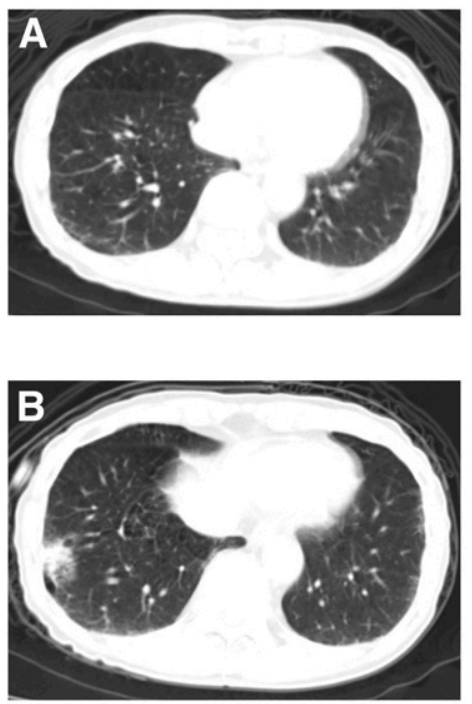
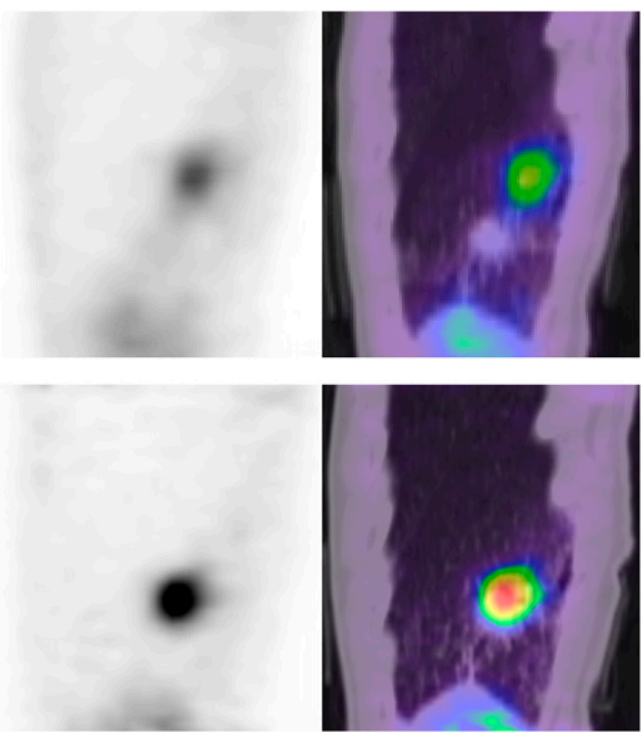

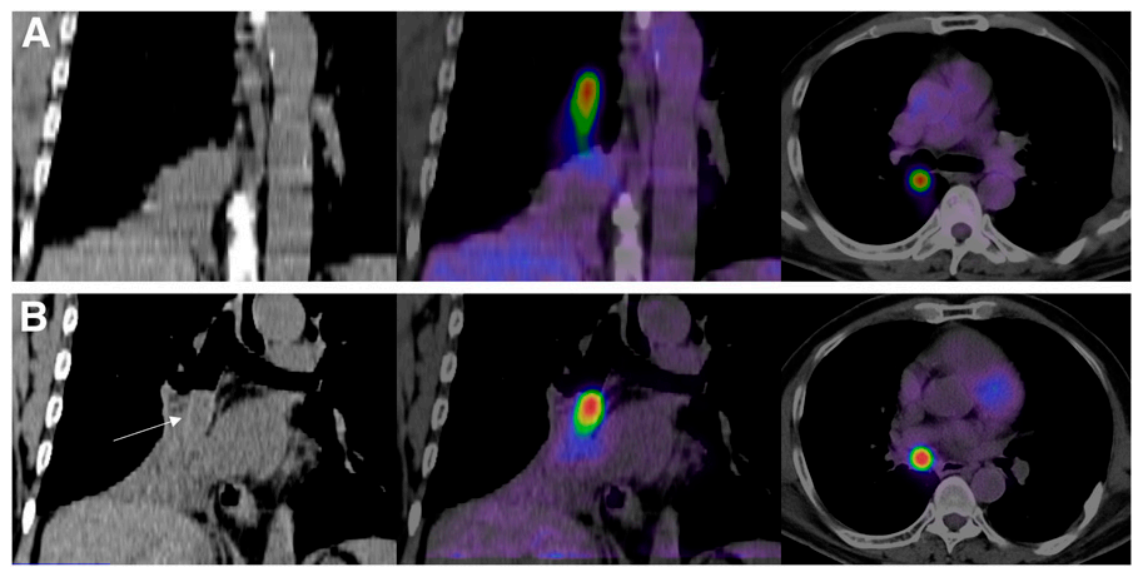

FIGURE 4. Coronal free-breathing CT and coronal and transaxial freebreathing PET/CT scans (A), as well as coronal breath-hold CT and coronal and transaxial breath-hold PET/ CT scans (B), of patient with lung cancer (arrow) in right main bronchus (squamous cell carcinoma, $20 \mathrm{~mm}$ ). Free-breathing SUVmax $=6.61$, breath-hold SUVmax $=23.02$, and difference in SUVmax $=248.3 \%$. This patient had dyspnea associated with obstructive atelectasis of right lower lobe. In free-breathing PET/CT, ${ }^{18} \mathrm{~F}-\mathrm{FDG}$ uptake of tumor was seen outside bronchus because of misregistration between PET and CT. For radiotherapy planning, neither CT nor free-breathing PET/CT could discriminate tumor tissue from atelectasis. Breath-hold PET/CT was useful for accurately indicating tumor margin.

Deep-inspiration breath-hold PET/CT has been shown to be clinically feasible $(5,6)$. Using this method, Nehmeh et al. demonstrated an increase of $32.5 \%$ in median tumor SUV (range, $4 \%-83 \%$ ) and an improvement in spatial matching between PET and CT images, compared with non-motion-corrected PET/CT (6). However, this technique requires summed multiple-breath-hold PET acquisitions in nine 20-s independent frames, and asking patients to hold their breath at a certain point in their respiratory cycle may cause highly variable results. Patients must be trained to ensure reproducibility. In our experience, even a single breath-hold for $20 \mathrm{~s}$ was not acceptable for older patients with underlying lung diseases such as emphysema or pulmonary fibrosis.

In the present study, the single deep-inspiration breathhold method during PET was applied to reduce the examination time. We found in the phantom study that at least a 20 -s acquisition was required to obtain radioactivity equal to that of a 120-s acquisition (within $100 \% \pm 5 \%$ ) when the sphere was larger than $13 \mathrm{~mm}$. Thus, we determined that a 20-s frame would be used for acquisition of the breath-hold PET portion of the clinical PET/CT study. Our data showed an increase of $39.5 \%$ in mean tumor SUVmax (range, $2.9 \%-248.3 \%$ ), as compared with the SUVmax measured on free-breathing PET scans. This finding is consistent with previous studies using breath-hold technique $(5,6)$. The effective radiation dose to patients from the additional breath-hold CT was 1.1-1.3 mSv. This dose was equivalent

\begin{tabular}{|c|c|c|c|}
\hline Parameter & $\begin{array}{l}\text { High difference } \\
\qquad(n=13)\end{array}$ & $\begin{array}{l}\text { Low difference } \\
\qquad(n=34)\end{array}$ & $P$ \\
\hline Tumor size $(\mathrm{cm})$ & $2.45 \pm 0.87$ & $3.21 \pm 1.22$ & 0.043 \\
\hline $\begin{array}{l}\text { Free-breathing } \\
\text { SUVmax }\end{array}$ & $7.74 \pm 6.12$ & $8.46 \pm 3.95$ & 0.373 \\
\hline Breath-hold SUVmax & $14.06 \pm 11.21$ & $10.18 \pm 4.80$ & 0.379 \\
\hline
\end{tabular}

to the $1.16 \mathrm{mSv}$ found in another breath-hold PET/CT study (10) and slightly lower than the average $1.35 \mathrm{mSv}$ found for a low-dose cine CT scan (11).

For many patients, respiratory motion of the base of the lung, adjacent to the diaphragm, exceeds that of the apical or central areas of the lung. In one study, when motion was about $10 \mathrm{~mm}$ or more, SUV changes on the order of $20 \%$ were measured (12). Therefore, lesions near the lung base should be analyzed carefully, since diaphragmatic motion could induce serious fusion errors and a remarkable variation in SUV during different respiratory phases. In our results, tumors in the lower lung field showed a significantly higher percentage difference in SUVmax than did those in the upper lung field (Table 3), indicating that diaphragmatic motion significantly reduces tumor SUV in free-breathing PET. In addition, lung tumors with a high difference in SUVmax were significantly smaller than those with a low difference in SUVmax $(P=0.043)$ (Table 4). Particularly in the lowerlung group, the difference in SUVmax was more significantly affected by tumor size $(P=0.0083)$ (Table 6$)$. Therefore, our technique may better quantitate tumor SUV, especially in the lower lung field and for small tumors $(>13 \mathrm{~mm})$, which are likely to be influenced by respiratory motion.

Over the last few years, PET/CT has been used to plan radiation therapy (13-15). Depending on its sensitivity and specificity, PET/CT with ${ }^{18} \mathrm{~F}-\mathrm{FDG}$ sometimes has been shown to influence selection of the target volume for various types of cancer, including lung cancer. Figure 4 shows a

TABLE 5. Upper-Lung Group: Comparison Between Group with High Difference in SUVmax and Group with Low Difference in SUVmax

\begin{tabular}{|lccc}
\hline & High difference & Low difference & \\
\hline \multicolumn{1}{|c|}{ Parameter } & $(n=4)$ & $(n=18)$ & $P$ \\
\hline Tumor size $(\mathrm{cm})$ & $2.78 \pm 1.36$ & $2.96 \pm 1.28$ & 0.831 \\
\hline $\begin{array}{l}\text { Free-breathing } \\
\text { SUVmax }\end{array}$ & $5.98 \pm 4.87$ & $7.28 \pm 3.82$ & 0.580 \\
\hline Breath-hold SUVmax & $9.03 \pm 7.06$ & $8.47 \pm 4.38$ & 0.932 \\
\hline
\end{tabular}


TABLE 6. Lower-Lung Group: Comparison Between Group with High Difference in SUVmax and Group with Low Difference in SUVmax

High difference Low difference
Parameter

Tumor size $(\mathrm{cm})$

Free-breathing

SUVmax

Breath-hold SUVmax $16.29 \pm 12.3212 .11 \pm 4.63 \quad 0.497$ patient with lung cancer in the right main bronchus, associated with obstructive atelectasis of the right lower lobe. Although neither CT nor free-breathing PET/CT could discriminate tumor tissue from atelectasis, breath-hold PET/ CT clearly indicated the tumor margin for radiotherapy planning. Thus, the single 20-s acquisition of deep-inspiration breath-hold PET/CT may be useful for treatment planning in such cases.

In patients with lung cancer, PET/CT is increasingly used for evaluating therapeutic response. Most physicians rely on changes in SUV measurements from the baseline study to evaluate response during treatment. In free-breathing PET/CT, however, inaccurate measurement of tumor SUV due to respiratory motion may overestimate or underestimate the real therapeutic response. In this clinical setting, improved SUV measurement through the breath-hold PET/ CT technique will allow a more reliable assessment of therapeutic response.

One limitation of this study was that lung tumors with weak ${ }^{18}$ F-FDG uptake (free-breathing SUVmax $<1.5$ ) or small size ( $<13 \mathrm{~mm}$ in diameter) had to be excluded because of the short acquisition time for breath-hold PET. Visualization of tumors with weak ${ }^{18} \mathrm{~F}-\mathrm{FDG}$ uptake, typically seen as a ground-glass appearance on CT images, was poor in breath-hold PET because of background noise and low radioactivity. As shown in Table 1 , the $\mathrm{CV}$ in the phantom study tended to be higher in smaller spheres and increased with shorter acquisitions. The CV was 35.50 for a $13-\mathrm{mm}$ sphere, 23.95 for a $17-\mathrm{mm}$ sphere, and 8.06 for a $22-\mathrm{mm}$ sphere in a 20-s scan. It is possible that the $\mathrm{CV}$, which reflects statistical noise in the region of interest, may contribute to an increased tumor SUVmax in breath-hold PET.

In a previous study of the single deep-inspiration breathhold method, the patients were asked to hold their breath during maximal inspiration for as long as possible during the PET scan (mean breath-hold time, 54.2 s) (10). Although the results were similar to those of other studies using breath-hold technique $(6,16)$, sick patients may not accept such a long period of deep-inspiration apnea. Recently, in a phantom study, PET images with acquisition times of 45, 60, and $120 \mathrm{~s}$ had significantly higher diagnostic accuracy than did PET images during $120 \mathrm{~s}$ of simulated free breathing (performed by moving the phantom), suggesting that at least a 45 -s breath-hold is required (17). Further development of the PET/CT scanner will reduce the acquisition time and enable a more clinically practical breath-hold technique.

\section{CONCLUSION}

We have proposed a single 20-s acquisition of breath-hold PET for PET/CT examinations of lung tumors. Our study showed that this method enabled more precise measurement of tumor ${ }^{18} \mathrm{~F}$-FDG uptake, especially in the lower lung field and for small tumors $(>13 \mathrm{~mm}$ ), which may be affected by respiratory motion. This technique is clinically feasible and requires only a minor increase in examination time.

\section{REFERENCES}

1. Nakamoto Y, Tatsumi M, Cohade C, Osman M, Marshall LT, Wahl RL. Accuracy of image fusion of normal upper abdominal organs visualized with PET/CT. Eur J Nucl Med Mol Imaging. 2003;30:597-602.

2. Osman MM, Cohade C, Nakamoto Y, Marshall LT, Leal JP, Wahl RL. Clinically significant inaccurate localization of lesions with PET/CT: frequency in 300 patients. J Nucl Med. 2003;44:240-243.

3. Pan T, Mawlawi O, Nehmeh SA, et al. Attenuation correction of PET images with respiration-averaged CT images in PET/CT. J Nucl Med. 2005;46:1481-1487.

4. Allen-Auerbach M, Yeom K, Park J, Phelps M, Czernin J. Standard PET/CT of the chest during shallow breathing is inadequate for comprehensive staging of lung cancer. J Nucl Med. 2006;47:298-301.

5. Meirelles GS, Erdi YE, Nehmeh SA, et al. Deep-inspiration breath-hold PET/ CT: clinical findings with a new technique for detection and characterization of thoracic lesions. J Nucl Med. 2007;48:712-719.

6. Nehmeh SA, Erdi YE, Meirelles GS, et al. Deep-inspiration breath-hold PET/CT of the thorax. J Nucl Med. 2007;48:22-26.

7. Nehmeh SA, Erdi YE, Pan T, et al. Four-dimensional (4D) PET/CT imaging of the thorax. Med Phys. 2004;31:3179-3186.

8. Nehmeh SA, Erdi YE, Pan T, et al. Quantitation of respiratory motion during 4D-PET/CT acquisition. Med Phys. 2004;31:1333-1338.

9. Wolthaus JW, van Herk M, Muller SH, et al. Fusion of respiration-correlated PET and CT scans: correlated lung tumour motion in anatomical and functional scans. Phys Med Biol. 2005;50:1569-1583.

10. Kawano T, Ohtake E, Inoue T. Deep-inspiration breath-hold PET/CT of lung cancer: maximum standardized uptake value analysis of 108 patients. $J$ Nucl Med. 2008;49:1223-1231.

11. Pan T, Mawlawi O, Luo D, et al. Attenuation correction of PET cardiac data with low-dose average CT in PET/CT. Med Phys. 2006;33:3931-3938.

12. Erdi YE, Nehmeh SA, Pan T, et al. The CT motion quantitation of lung lesions and its impact on PET-measured SUVs. J Nucl Med. 2004;45:1287-1292.

13. Leong T, Everitt C, Yuen K, et al. A prospective study to evaluate the impact of FDG-PET on CT-based radiotherapy treatment planning for oesophageal cancer. Radiother Oncol. 2006;78:254-261.

14. Schwartz DL, Ford E, Rajendran J, et al. FDG-PET/CT imaging for preradiotherapy staging of head-and-neck squamous cell carcinoma. Int J Radiat Oncol Biol Phys. 2005;61:129-136.

15. van Der Wel A, Nijsten S, Hochstenbag M, et al. Increased therapeutic ratio by ${ }^{18}$ FDG-PET CT planning in patients with clinical CT stage N2-N3M0 non-smallcell lung cancer: a modeling study. Int J Radiat Oncol Biol Phys. 2005;61:649655.

16. Fin L, Daouk J, Morvan J, et al. Initial clinical results for breath-hold CT-based processing of respiratory-gated PET acquisitions. Eur J Nucl Med Mol Imaging. 2008;35:1971-1980.

17. Yamaguchi $\mathrm{T}$, Ueda $\mathrm{O}$, Hara $\mathrm{H}$, et al. Usefulness of a breath-holding acquisition method in PET/CT for pulmonary lesions. Ann Nucl Med. 2009;23:65-71. 